\title{
Computed tomography coronary angiography vs. standard diagnostic procedure for the diagnosis of angina due to coronary heart disease: A cross-sectional study
}

\author{
ZHENG WU ${ }^{1}$, YI HE ${ }^{2}$, WENZHENG $\mathrm{LI}^{1}$ and SHUJUAN CHENG ${ }^{1}$ \\ Departments of ${ }^{1}$ Cardiology and ${ }^{2}$ Radiology, Beijing Anzhen Hospital, Capital Medical University, \\ Beijing Institute of Heart, Lung and Blood Vessel Diseases, Beijing 100029, P.R. China
}

Received July 29, 2018; Accepted December 6, 2018

DOI: $10.3892 /$ etm.2019.7229

\begin{abstract}
Patients with episodes of angina are likely to experience future cardiac events and benefit from a revascularization procedure. Conventional invasive coronary angiography is a well-established and reliable method for the detection of angina, but it has a risk of complications and false-negative diagnosis. The objective of the present study was to assess the utility of computed tomography coronary angiography (CTCA) in the diagnosis of angina due to coronary heart disease. A total of 2,426 patients with chest pain referred to the rapid access chest pain clinic of Beijing Anzhen Hospital, Capital Medical University (Beijing, P.R. China) between 18 January 2016 and 1 December 2017 were included in the present cross-sectional study. All patients were subjected to evaluation of symptoms, blood tests, 12-lead electrocardiogram (ECG), exercise ECG, coronary artery calcium scoring and CTCA. The cost of the diagnosis of angina was determined for each individual method. In total, $776(32 \%)$ and $1,420(58 \%)$ of patients were identified to be abnormal on clinical assessment and CTCA, respectively. Exercise ECG results were not correlated with the interpretation of CTCA $(\mathrm{r}=0.8511)$. The working area of the angina due to coronary heart disease detected at one time by the different diagnostic procedures was in the order of ECG <clinical assessment $<$ exercise ECG < coronary artery calcium scoring <CTCA. The cost of the 'standard diagnostic procedure (clinical assessments, ECG, exercise ECG)’ was 15,452 $\pm 806 ¥ /$ patient and that of CTCA was $12,546 \pm 612 ¥ /$ patient. CTCA had a higher sensitivity for the diagnosis of angina due to coronary heart disease and the cost was lower than that of the "standard
\end{abstract}

Correspondence to: Dr Shujuan Cheng, Department of Cardiology, Beijing Anzhen Hospital, Capital Medical University, Beijing Institute of Heart, Lung and Blood Vessel Diseases, 2 Anzhen Road, Beijing 100029, P.R. China

E-mail: shujuancheng684@hotmail.com

Key words: angina, computed tomography, coronary angiography, coronary heart disease, standard diagnostic procedure diagnostic procedure' (level of evidence: 3). The current study was registered at the Research Registry on 11th January 2016 (trial no. researchregistry4232).

\section{Introduction}

Patients with episodes of angina are likely to experience cardiac events in the next two years (1) and benefit from undergoing a revascularization procedure (2). Clinical research is required to be performed to improve the assessment of the cause of chest pain in patients with suspected angina to reduce the risk of cardiovascular events (3). At present, misdiagnosis occurs frequently; for instance, individuals presenting with chest pain diagnosed with non-cardiac conditions account for one-third of cases diagnosed with cardiovascular disease in the follow-up time (false-negative diagnosis of angina) (4). Therefore, diagnosis and risk assessments require improvements to be reduce the rate of false negative diagnosis (5).

In general, any patient referred to a cardiologist with complaints of chest pain is subjected to 12-lead electrocardiogram (ECG) to detect the possible presence of angina (6). Further diagnostic assessment includes exercise ECG (7). In addition, conventional invasive coronary angiography is a well-established and reliable method for the detection of angina due to coronary heart disease, but this method is not recommended in such patients due to contraindications, including access site problems, severe allergies to intravenous contrast agents, myocardial infarction, arrhythmia and stroke (8).

National Institute for Health and Care Excellence clinical guidelines recommend the use of Computed Tomography Coronary Angiography (CTCA) for acute chest pain (9). For the detection of coronary cardiac disease(s), considering invasive coronary angiography is the reference standard, CTCA has $>95 \%$ specificity, $>85 \%$ sensitivity and acceptable positive (range, $>93$ and $81 \%$ ) and negative predictive rates (range, $<2$ and 9\%) (8). Individuals with an arrhythmia, obesity and/or coronary calcification, the image quality in CTCA is poor (10). CTCA is associated with a high radiation (20 mSv) exposure, which may induce cancer. Although the radiation dose may be reduced to $2 \mathrm{mSv}$, a reduction of the radiation dose always hampers the image quality (11). The diagnostic cost and duration of hospital stay for an individual receiving CTCA are less 
than those of the 'standard diagnostic procedure' (12). However, due to limited availability of trained hospital staff and medical equipment at the Beijing Anzhen Hospital, Capital Medical University (Beijing, China), it is challenging to offer CTCA to all patients according to the National Institute for Health and Care Excellence clinical guidelines.

The primary aim of the present study was to compare the detection of angina due to coronary heart disease using CTCA (CTCA and coronary artery calcium scoring) with the "standard diagnostic procedure' (clinical assessments, ECG and exercise ECG) in Chinese patients with chest pain referred to the rapid access chest pain clinic of Beijing Anzhen Hospital, Capital Medical University (Beijing, China). The secondary endpoint of the study was to investigate the sensitivity and accuracy of CTCA with regard to the 'standard diagnostic procedure' at a level of evidence of 3 without any conflict of interest.

\section{Materials and methods}

Ethical approval and consent to participate. The present study was registered in the research registry (www.researchregistry. com; unique identifying no. 4232; date, 11 January 2016). The protocol (no. BAH/CL/01/16 dated 15 December 2015) was approved by the review board of Beijing Anzhen Hospital (Beijing, China). An informed consent form regarding radiological images, pathology, clinical assessments and publication of patient data and personal images (if any) in all formats (hard copy and/or electronic format) irrespective of time and language had been signed by all patients enrolled or their relatives (legally authorized guardian). The present study adhered to the law of China, the Standards for the Reporting of Diagnostic Accuracy Studies (STARD) guidelines from 2015 (13) and the 2013 version of the Declaration of Helsinki (14).

Inclusion criteria. Patients aged $\geq 18$ years were included in the current study if written informed consent was provided. Each patient completed a patient information sheet on arrival at the hospital (15). On the basis of the information provided, patients with chest pain and suspected angina due to coronary heart disease(s), referred (by medical officer of emergency department and/or whole-body check-up department) to the rapid access chest pain clinic of Beijing Anzhen Hospital, Capital Medical University (Beijing, China) between 18 January 2016 and 1 December 2017, were included in the study. Demographic characteristics of all of the enrolled patients are provided in Table I.

Exclusion criteria. Patients who had refused to undergo CTCA, and those with acute coronary syndrome (up to 100 days previously), chronic kidney failure (or glomerular filtration rate $\leq 29.8 \mathrm{ml} / \mathrm{min}$ or serum creatinine $\geq 251 \mu \mathrm{M} / \mathrm{l}$ ) and female patients with pregnancies were excluded from the current study. The patients who were greater than the maximum height and weight range of the scanner were excluded from the study. Patients with confirmed angina or high-density lipoprotein (HDL) levels of $<20 \mathrm{mg} / \mathrm{dl}$ and total cholesterol of $>600 \mathrm{mg} / \mathrm{dl}$ were excluded from the current study and subjected to treatment directed by a cardiologist (16). Patients with gastroesophageal reflux, gastroesophageal disease and
Table I. Demographic characteristics of the subjects $(n=2,426)$.

\begin{tabular}{lc}
\hline Characteristics & Value \\
\hline Age (years) & $63.36 \pm 10.55$ \\
Gender & \\
Male & $1,457(60)$ \\
Female & $969(40)$ \\
Smoking & \\
Currently & $480(20)$ \\
Previously & $505(21)$ \\
Duration of chest pain (months) & \\
$<1$ & $1,189(49)$ \\
$1-6$ & $775(32)$ \\
$6-12$ & $265(11)$ \\
$>12$ & $197(8)$ \\
Body height (cm) & $161.09 \pm 3.89$ \\
Body weight (kg) & $61.02 \pm 4.54$ \\
Body mass index (kg/m²) & $23.55 \pm 2.1$ \\
Arthritis & $218(9)$ \\
Chronic obstructive pulmonary disease & $314(13)$ \\
and/or asthma & \\
Neuro and/or psychological deficits & \\
Cancer & $194(8)$ \\
Abnormality in the gastrointestinal tract & $72(3)$ \\
Family history of angina due to coronary & $96(4)$ \\
heart disease & $479(19)$ \\
Rural residence & \\
Income quintile & $602(25)$ \\
1 (lowest) & \\
2 (low) & $645(23)$ \\
3 (moderate) & \\
4 (high) & \\
\hline (highest) & \\
\hline
\end{tabular}

All enrolled patients were of Chinese origin. Values are expressed as the mean \pm standard deviation or $\mathrm{n}(\%)$.

diseases that are easily mistaken as coronary heart disease were excluded from the study.

Experimental design. A total of 2,426 patients were included in the present cross-sectional study. The STARD flow diagram of the study is presented in Fig. 1. Patients had undergone a clinical assessment with CTCA (16). At the rapid access chest pain clinic of Beijing Anzhen Hospital, all patients were subjected to a normal clinical assessment as outlined below subsequent to enrollment.

\section{Clinical assessment}

Determination of symptoms. Substernal chest pain provoked by emotional stress/exertion and relieved by rest and/or nitroglycerin treatment was defined as typical angina. Pain in the center of the chest that was not associated with the heart, and accompanied with sleepiness, weight gain, increased appetite, 


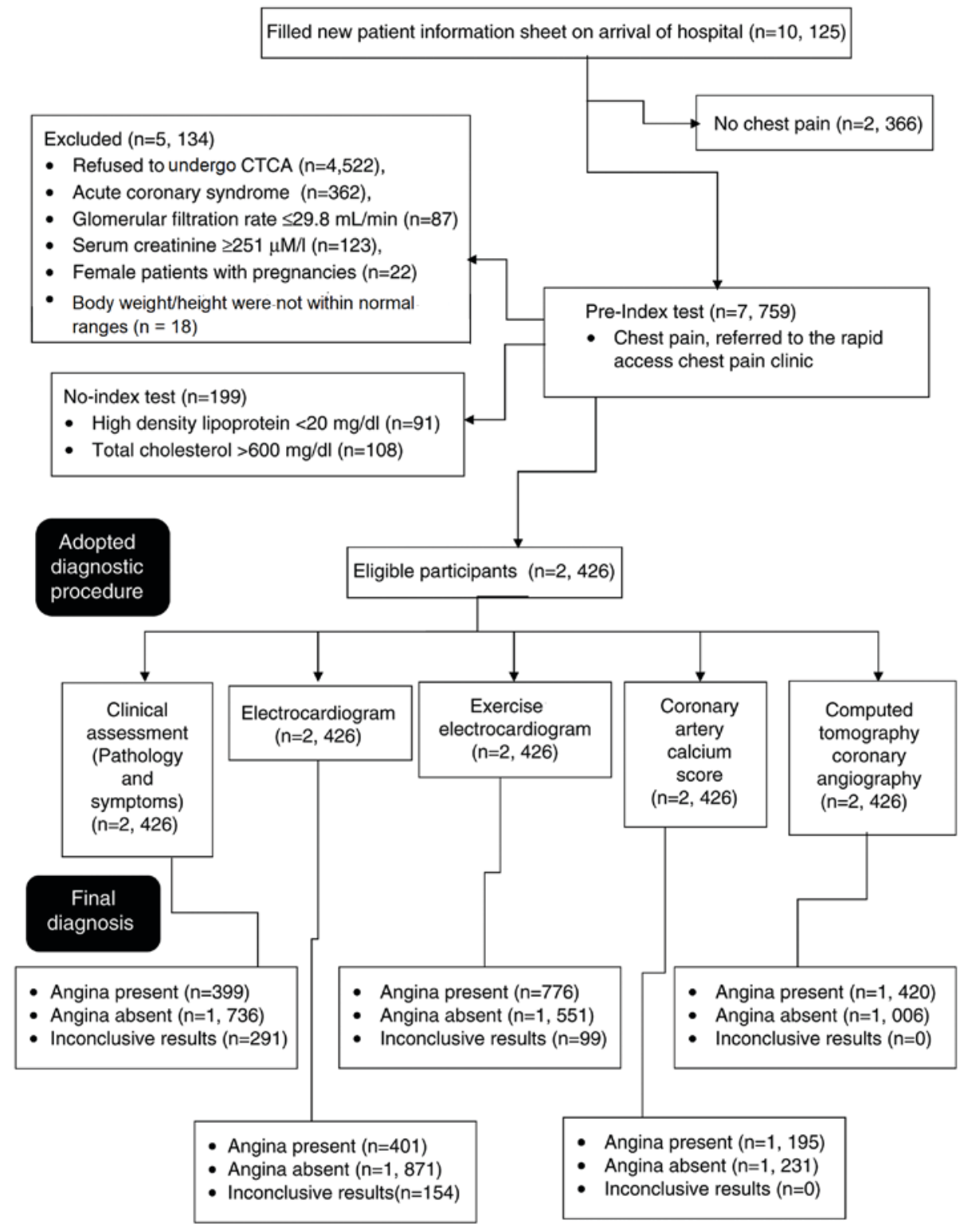

Figure 1. Flow diagram of diagnostic procedures adopted for possible angina according to the Standards for the Reporting of Diagnostic Accuracy Studies. CTCA, computed tomography coronary angiography.

excessive sleep, mood changes, fatigue and/or weakness, and that was not relieved by rest and/or nitroglycerin was defined as atypical angina. Esophageal spasm pain and/or cervical root compression pain that was relieved by rest and/or nitroglycerin was defined as non-anginal pain (17).

The blood pressure was measured with a sphygmomanometer (Omron 8712; Omron Healthcare, Jakarta, Indonesia). Hypertension was defined as a diastolic blood pressure of $>90 \mathrm{mmHg}$ and a systolic blood pressure of $>140 \mathrm{mmHg}(18)$.

Blood tests. A blood sample was collected from all patients and subjected to detection of total cholesterol, low-density lipoprotein (LDL), HDL, blood serum creatinine, random plasma glucose levels, hemoglobin, the percentage of glycated hemoglobin (normal value, 6\%) and troponin serum levels (normal value, $0.49 \mathrm{ng} / \mathrm{ml}$ ) (16). Diabetes was defined as random plasma glucose of $>160 \mathrm{mg} / \mathrm{dl}$. Hyperlipidemia was defined as total cholesterol levels of $>240 \mathrm{mg} / \mathrm{dl}$, LDL of $>160 \mathrm{mg} / \mathrm{dl}$ and/or HDL of $<40 \mathrm{mg} / \mathrm{dl}$ (18).

$E C G$. The chest, arms and lower legs were exposed and electrodes were positioned, 12-lead ECG and exercised-ECG were performed according to the Bruce protocol (16). If the heart was beating in a regular sinus rhythm in the range of 60-100 (specifically 82 ) beats per minute and waves and intervals had normal values [RR-interval, 0.6-1.2 sec; P-wave, $80 \mathrm{msec}$, PR-interval, 120-200 msec; PR-segment, 50-120 msec; QRS-complex, 80-100 msec; ST segment, 80-120 msec; and T-wave, $160 \mathrm{msec}(17)]$ were considered as normal ECG. The Duke treadmill score was calculated according to the following equation: Duke treadmill score=Exercise time-(5x ST segment deviation in mm-(4x angina index score). Grading was performed according to the Duke treadmill score with a score of $\geq 5$ indicating a low risk, -10 to +4 intermediate risk and $\leq-11$ high risk of further cardiac events (19). 
Table II. Non-zero calcium score probability estimation for a Chinese population.

\begin{tabular}{|c|c|c|c|c|c|c|c|c|}
\hline \multirow[b]{2}{*}{ Age (years) } & \multicolumn{4}{|c|}{ Males } & \multicolumn{4}{|c|}{ Females } \\
\hline & 25 th & 50th & 75 th & 90th & 25 th & 50th & 75 th & 90th \\
\hline$\leq 45$ & 0 & 0 & 3 & 50 & 0 & 0 & 0 & 0 \\
\hline 46 & 0 & 0 & 4 & 56 & 0 & 0 & 0 & 0 \\
\hline 47 & 0 & 0 & 6 & 63 & 0 & 0 & 0 & 0 \\
\hline 48 & 0 & 0 & 8 & 71 & 0 & 0 & 0 & 2 \\
\hline 49 & 0 & 0 & 11 & 80 & 0 & 0 & 0 & 6 \\
\hline 50 & 0 & 0 & 14 & 89 & 0 & 0 & 0 & 12 \\
\hline 51 & 0 & 0 & 17 & 98 & 0 & 0 & 0 & 17 \\
\hline 52 & 0 & 0 & 21 & 110 & 0 & 0 & 0 & 23 \\
\hline 53 & 0 & 0 & 25 & 124 & 0 & 0 & 0 & 30 \\
\hline 54 & 0 & 0 & 29 & 136 & 0 & 0 & 1 & 38 \\
\hline 55 & 0 & 0 & 34 & 150 & 0 & 0 & 2 & 47 \\
\hline 56 & 0 & 0 & 38 & 163 & 0 & 0 & 5 & 57 \\
\hline 57 & 0 & 0 & 43 & 176 & 0 & 0 & 7 & 66 \\
\hline 58 & 0 & 1 & 49 & 194 & 0 & 0 & 10 & 77 \\
\hline 59 & 0 & 3 & 58 & 215 & 0 & 0 & 14 & 90 \\
\hline 60 & 0 & 5 & 67 & 242 & 0 & 0 & 18 & 105 \\
\hline 61 & 0 & 7 & 279 & 273 & 0 & 0 & 23 & 122 \\
\hline 62 & 0 & 10 & 91 & 304 & 0 & 0 & 28 & 144 \\
\hline 63 & 0 & 13 & 102 & 329 & 0 & 0 & 34 & 160 \\
\hline 64 & 0 & 15 & 112 & 350 & 0 & 0 & 39 & 178 \\
\hline 65 & 0 & 18 & 121 & 372 & 0 & 0 & 45 & 194 \\
\hline 66 & 0 & 21 & 132 & 397 & 0 & 0 & 51 & 211 \\
\hline 67 & 0 & 24 & 143 & 427 & 0 & 0 & 55 & 220 \\
\hline 68 & 0 & 28 & 154 & 450 & 0 & 2 & 59 & 229 \\
\hline 69 & 0 & 32 & 166 & 470 & 0 & 3 & 64 & 235 \\
\hline 70 & 0 & 34 & 174 & 487 & 0 & 5 & 70 & 243 \\
\hline 71 & 0 & 37 & 183 & 503 & 0 & 7 & 76 & 262 \\
\hline 72 & 0 & 40 & 191 & 522 & 0 & 9 & 83 & 276 \\
\hline 73 & 1 & 45 & 201 & 546 & 0 & 11 & 89 & 287 \\
\hline 74 & 2 & 49 & 216 & 570 & 0 & 13 & 96 & 300 \\
\hline 75 & 3 & 53 & 229 & 599 & 0 & 16 & 103 & 314 \\
\hline 76 & 5 & 58 & 241 & 629 & 0 & 18 & 111 & 332 \\
\hline 77 & 6 & 63 & 254 & 659 & 0 & 22 & 119 & 347 \\
\hline 78 & 8 & 70 & 273 & 695 & 0 & 25 & 128 & 361 \\
\hline 79 & 9 & 75 & 288 & 735 & 0 & 28 & 137 & 377 \\
\hline 80 & 11 & 81 & 305 & 769 & 0 & 32 & 146 & 398 \\
\hline 81 & 13 & 88 & 325 & 808 & 2 & 35 & 158 & 416 \\
\hline 82 & 15 & 95 & 344 & 855 & 3 & 40 & 167 & 436 \\
\hline 83 & 18 & 103 & 369 & 913 & 5 & 44 & 177 & 456 \\
\hline$\geq 84$ & 20 & 112 & 391 & 971 & 6 & 50 & 190 & 483 \\
\hline
\end{tabular}

This table was obtained from Multi-Ethnic Study of Atherosclerosis (https://www.mesa-nhlbi.org/Calcium/input.aspx).

Coronary artery calcium score. All enrolled patients were subjected to a scan of the heart by electron-beam CT (Philips Healthcare, Eindhoven, The Netherlands). Radiological images were analyzed by experts (radiologists with a minimum three years of experience in image analysis) using Multi-Ethnic Study of Atherosclerosis software (Collaborative Health
Studies Coordinating Center, Seattle, WA, USA) to determine the Agatston score and the presence of calcium (18). The normal value of the calcium score percentile with regard to age was estimated (Table II). The Agatston score and the presence of calcium were interpreted by a radiologist $(\mathrm{YH})$ who had at least three years of experience. 
Table III. Interpretation of computed tomography coronary angiography and coronary artery calcium score.

Observation

\begin{tabular}{|c|c|c|c|}
\hline \multirow[b]{2}{*}{ Location } & \multirow{2}{*}{$\begin{array}{c}\text { Degree of } \\
\text { stenosis }(\%)\end{array}$} & \multicolumn{2}{|r|}{ Interpretation } \\
\hline & & Stenosis & Angina due to heart disease \\
\hline \multirow{4}{*}{$\begin{array}{l}\text { Luminal cross-sectional } \\
\text { area of at least } 1 \text { major } \\
\text { epicardial vessel }\end{array}$} & $<10$ & Normal & No or minimal coronary artery disease \\
\hline & $10-49$ & $\begin{array}{l}\text { Hemodynamically } \\
\text { insignificant }\end{array}$ & $\begin{array}{l}\text { Non-obstructive coronary artery disease/atherosclerotic } \\
\text { plaque }\end{array}$ \\
\hline & $50-69$ & Intermediate & $\begin{array}{l}\text { Moderate non-obstructive coronary artery disease/ } \\
\text { atherosclerotic plaque }\end{array}$ \\
\hline & $\geq 70$ & Significant & Obstructive coronary artery disease/atherosclerotic plaque \\
\hline Left main stem & $\geq 50$ & Significant & $\begin{array}{l}\text { Mild non-obstructive coronary artery disease/atherosclerotic } \\
\text { plaque }\end{array}$ \\
\hline Total/subtotal occlusion & 100 & Significant & Obstructive coronary artery disease/atherosclerotic plaque \\
\hline $\begin{array}{l}\text { Calcium score }>400 \\
\text { Agatston units }\end{array}$ & Inconclusive & Inconclusive & $\begin{array}{l}\text { Non-obstructive coronary artery disease/atherosclerotic } \\
\text { plaque }\end{array}$ \\
\hline $\begin{array}{l}\text { Calcium score } 90 \text { th } \\
\text { percentile for sex and age }\end{array}$ & Inconclusive & Inconclusive & $\begin{array}{l}\text { Non-obstructive coronary artery disease/atherosclerotic } \\
\text { plaque }\end{array}$ \\
\hline
\end{tabular}

CTCA. CTCA was performed using a 256-slice CT scanner (Brilliance iCT; Philips Healthcare) by using the single breath-hold protocol. Patients with a systolic blood pressure of $>110 \mathrm{mmHg}$ and a heart rate of $>60$ beats $/$ min received $0.5 \mathrm{mg}$ sublingual glyceryl trinitrate (Angised; Glaxo Smith Kline Pharmaceuticals Ltd., Beijing, China) prior to CTCA (16). Coronary angiograms were interpreted by clinicians (WZ, WL and SC) with at least three years of experience using the scanner's workstation. In the case of a disagreement between the clinicians, a consensus regarding the revascularization procedure was reaching following an interpretation by a cardiologist with at least three years of experience. Significant stenosis and angina due to heart disease were defined as per Table III (20).

The benefits score (the difference between the possible benefit and the possible harm associated with each procedure following the revascularization procedure) of diagnostic modalities were evaluated by decision curve analysis according to the following equation (21):

$$
\mathrm{BS}=\frac{\mathrm{A}}{\mathrm{C}}-\left(\frac{\mathrm{B}}{\mathrm{C}} \times \frac{\mathrm{D}}{1-\mathrm{D}}\right),
$$

where BS is the benefit score of the adopted diagnostic procedure for the detection of angina due to coronary heart disease, $\mathrm{A}$ is the number of individuals with accurate detection of angina, $\mathrm{B}$ is the number of individuals with no accurate detection of angina, $\mathrm{C}$ is the total number of individuals subjected to the procedure and $\mathrm{D}$ is the level of diagnostic confidence; above this level, the revascularization procedure could be recommended.

Cost. The cost of the diagnosis included the cost of emergency department and/or whole-body check-up department utilization, the cost of diagnostic modalities and pathology, and expert charges (12).
Statistical analysis. InStat software (GraphPad Software Inc., La Jolla, CA, USA) was used for statistical analysis. One-way analysis of variance was performed to compare results and cost between the 'standard diagnostic procedure' and CTCA (22). Pearson correlation analysis [considering the Pearson coefficient ( $\mathrm{r}$ ) in the range of $0.8543-0.8617$ as significant] was performed to determine the possible correlation between exercise ECG results and interpretation of CTCA (23). $\mathrm{P}<0.01$ was considered to indicate statistical significance.

\section{Results}

Clinical assessment. In total, 776 patients (32\%) were identified to be abnormal on clinical assessment. According to anginal symptoms, 748 patients had non-anginal pain, 399 had typical angina and 988 had atypical angina. By contrast, 12-lead ECG concluded that 401 (17\%) patients were abnormal. Exercised-ECG concluded that $356(15 \%)$ patients had a low risk, 266 (11\%) patients had an intermediate risk and $154(6 \%)$ patients had a high risk of further cardiac events (Table IV). A total of 1,420 patients (58\%) were considered abnormal according to their coronary artery calcium score and CTCA. CTCA concluded that $658(27 \%)$ patients had obstructive and $762(31 \%)$ had non-obstructive coronary artery disease (Table V). The coronary artery calcium score and CTCA had a higher sensitivity regarding the diagnosis of angina due to coronary heart disease compared with the 'standard diagnostic procedure' $(\mathrm{P}<0.0001)$.

ECG results. The results of the 12-lead ECG were only considered for patients with typical angina (Fig. 2), while for asymptomatic patients, ECGs were normal or did not have any predictive value regarding angina (Fig. 3). The exercise ECG 
Table IV. Results of the standard diagnostic procedure diagnostic procedure in the cohort $(n=2,426)$.

\begin{tabular}{|c|c|}
\hline Characteristics & Value \\
\hline Diabetes $^{\mathrm{a}}$ & $515(21)$ \\
\hline Hypertension $^{\mathrm{b}}$ & $1,119(46)$ \\
\hline Hyperlipidemia $^{c}$ & $848(35)$ \\
\hline Hemoglobin (g/dl) & $13.88 \pm 1.89$ \\
\hline $\operatorname{HbA1c} c^{d}(\%)$ & $6.15 \pm 1.31$ \\
\hline Serum creatinine $^{\mathrm{e}}(\mu \mathrm{M} / \mathrm{l})$ & $215.58 \pm 4.55$ \\
\hline Troponin serum level ${ }^{\mathrm{f}}(\mathrm{ng} / \mathrm{ml})$ & $0.39 \pm 0.01$ \\
\hline \multicolumn{2}{|l|}{ Anginal symptoms } \\
\hline Non-anginal & $748(31)$ \\
\hline Typical & 399 (16) \\
\hline Atypical & $988(41)$ \\
\hline Inconclusive results & $291(12)$ \\
\hline \multicolumn{2}{|l|}{12 lead-ECG } \\
\hline Normal & $1871(77)$ \\
\hline Abnormal & 401 (17) \\
\hline Inconclusive results & $154(6)$ \\
\hline \multicolumn{2}{|l|}{ Exercise ECG } \\
\hline Normal $^{\mathrm{g}}$ & $1,551(64)$ \\
\hline \multicolumn{2}{|l|}{ Abnormal $^{\mathrm{h}}$} \\
\hline Low risk & $356(15)$ \\
\hline Intermediate risk & $266(11)$ \\
\hline High risk & $154(6)$ \\
\hline Inconclusive results & $99(4)$ \\
\hline
\end{tabular}

Values are expressed as the mean \pm standard deviation or $\mathrm{n}(\%)$. ${ }^{\mathrm{a}}$ Normal values for blood glucose, $<160 \mathrm{mg} / \mathrm{dl}$ (random). ${ }^{\mathrm{b}}$ Normal blood pressure, 140/90 mmHg. 'Normal values for total cholesterol, $<240 \mathrm{mg} / \mathrm{dl}$; low-density lipoprotein, $<160 \mathrm{mg} / \mathrm{dl}$; and high-density lipoprotein, $>40 \mathrm{mg} / \mathrm{dl}$. "Normal value, $6 \%$. 'Normal value, $<251 \mu \mathrm{M} / 1$. ${ }^{\mathrm{f}}$ Normal value, $0.49 \mathrm{ng} / \mathrm{ml}$; ${ }^{\mathrm{g}}$ Sinus rhythm in the range of

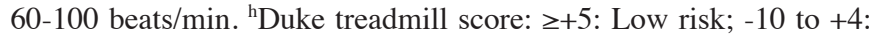
Intermediate risk; $\leq-11$ : High risk. ECG, electrocardiogram; HbA1c, glycated hemoglobin.

results were not correlated with the interpretation of CTCA $(r=0.8511$; data not shown).

Cost. The cost of the 'standard diagnostic procedure' was $15,452 \pm 806 ¥$ patient and that of CTCA with coronary artery calcium scoring was $12,546 \pm 612 ¥ /$ patient (Fig. 4).

The benefits score. The working area of the diagnosis that detected angina due to coronary heart disease at one time among the adopted diagnostic procedures was in the order of ECG <clinical assessments <exercised ECG <coronary artery calcium score $<$ CTCA. Clinical assessment followed by 12-lead ECG and exercise ECG had a level of confidence of only $5-35 \%$ for a revascularization procedure and at $>35 \%$, there was a risk of overdiagnosis. However, CTCA with the coronary artery calcium score had a level of confidence of $5-64 \%$ for the revascularization procedure (Fig. 5).

\section{Discussion}

The present large-population study compares the use of CTCA and the standard diagnostic procedure for diagnosing patients presenting with chest pain with angina due to coronary heart disease at an emergency unit specialized in Beijing Anzhen Hospital, Capital Medical University. The standard diagnostic procedure for the diagnosis is invasive and had more inconclusive results (291 for anginal symptoms, 154 for ECG and 99 for exercise ECG) than CTCA. If CTCA is unavailable to subjects with inconclusive results of the standard diagnostic procedure for the diagnosis of chest pain, the appropriate treatment is elusive and the risk of cardiovascular events is increased $(3,24)$. In such cases, conventional CA may be performed; however, it is an invasive procedure, with more complications and higher chances of a false-negative diagnosis compared with CTCA (25). However, CTCA is a non-invasive method of diagnosis and may be used to spare patients from further stressful and invasive testing for angina (26). With respect to the results of CTCA, it is a most desirable diagnostic modality for angina due to coronary heart disease.

The present study analyzed the benefit of imaging modalities by assessing anatomic testing vs. functional testing. However, previous studies compare patient-centered and clinician-centered outcomes $(16,18,26)$. Furthermore, certain studies reported on randomized controlled trials adhering to the Consolidated Standards of Reporting Trials guidelines with total populations sizes of 3,427 [sample size (n); 1,755 vs. 1,672; CTCA vs. standard diagnostic procedure] (1), 562 (n, 332 vs. 240) (12), 4,138 (n, 2,069 vs. 2,069) (16) and 4,146 (n, 2,073 vs. 2,073) $(26,27)$, and the studies were performed with 6-month follow-up periods with medication(s). Of note, in these previous studies, the diagnostic methods were used for initial diagnosis and diagnostic data were evaluated from a non-treatment randomization design; however, it may not be possible to evaluate the sensitivity and accuracy of any diagnostic method using two groups, as the demographic parameters would be different between the two groups, and randomization may only be appropriate for treatment studies (28). If these studies were still considered to be randomized trials, it may be difficult to determine the phase, e.g. Phase I (on healthy volunteers; sample size, 20-100), Phase II (diseased patients; sample size, 100-300) or Phase III (diseased patients; sample size, 300-3,000) (29); according to the sample size, these studies do not meet the criteria for any randomized drug trial. Furthermore, the role of diagnostic modalities in the initial diagnosis and during follow-up periods of medication(s) is not clarified in these studies. With regard to the design of the present study, the study provided an exact comparison of CTCA and the 'standard diagnostic procedure' for the diagnosis of angina due to coronary heart disease.

In $4 \%$ of subjects, the ECG results were inconclusive. However, CTCA provided information on obstructive (27\%) and non-obstructive (31\%) coronary artery disease, as also reported previously (26). With respect to the specificity of diagnostic modalities adopted by clinicians for the diagnosis of patients with suspected angina, the standard diagnostic procedure underestimated the possible risk of cardiac events in patients with chest pain. 
Table V. Results of coronary artery calcium score and computed tomography coronary angiography in the cohort $(\mathrm{n}=2,426)$.

Characteristic

Interpretation of age- and sex-dependent coronary artery calcium score

No or minimal coronary artery disease

Non-obstructive coronary artery disease/atherosclerotic plaque

Interpretation of computed tomography coronary angiography

No or minimal coronary artery disease

Non-obstructive coronary artery disease/atherosclerotic plaque

Mild

Moderate

Number of vessels with obstructive coronary artery disease/atherosclerotic plaque

1

3

$106(4)$

Other cardiac-associated findings

Dilated right atrium

Aortic valve calcification

Left ventricular wall thinning

Mitral valve calcification

Left ventricular hypertrophy

Dilated left ventricle

Hypertrophic obstructive

Dilated right ventricle

Cardiomyopathy

Dilated left atrium

Non-cardiac findings

Parenchymal lung disease

Liver pathology

Pulmonary mass or nodule

Lymphadenopathy

Emphysema

Pulmonary embolism

Hiatus hernia

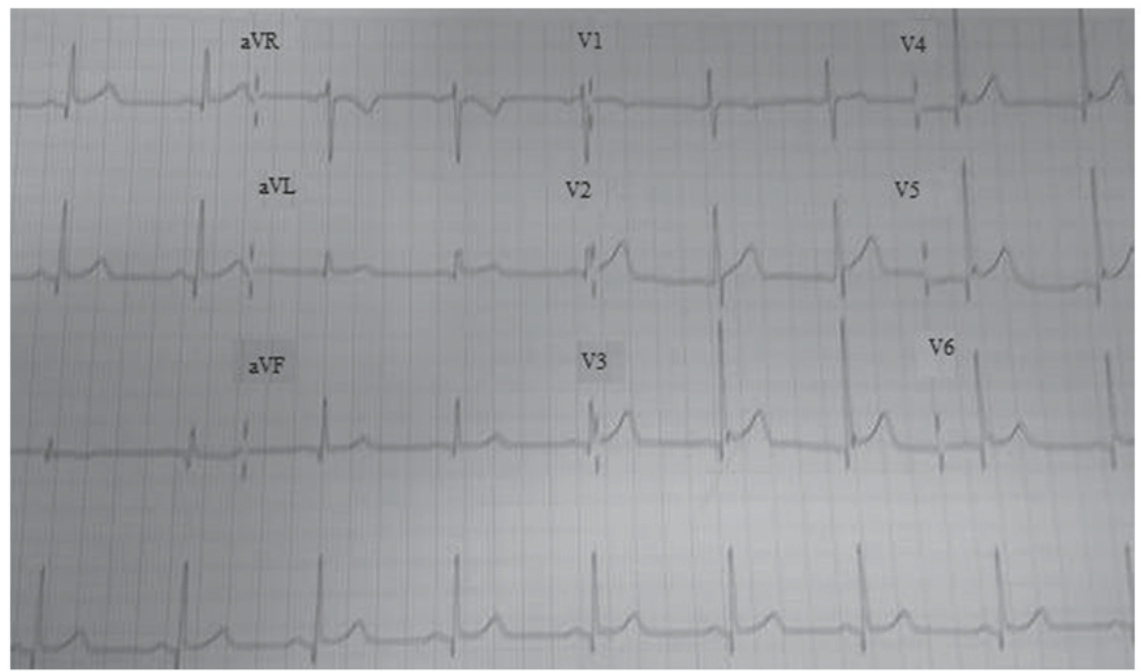

Figure 2. 12-leads electrocardiogram of a patient with angina (age, 30 years). I, II, III, V1, V2, V3, V4, V5 and V6 are external leads. aVR, augmented vector right; aVL, augmented vector left; aVF, augmented vector foot. 


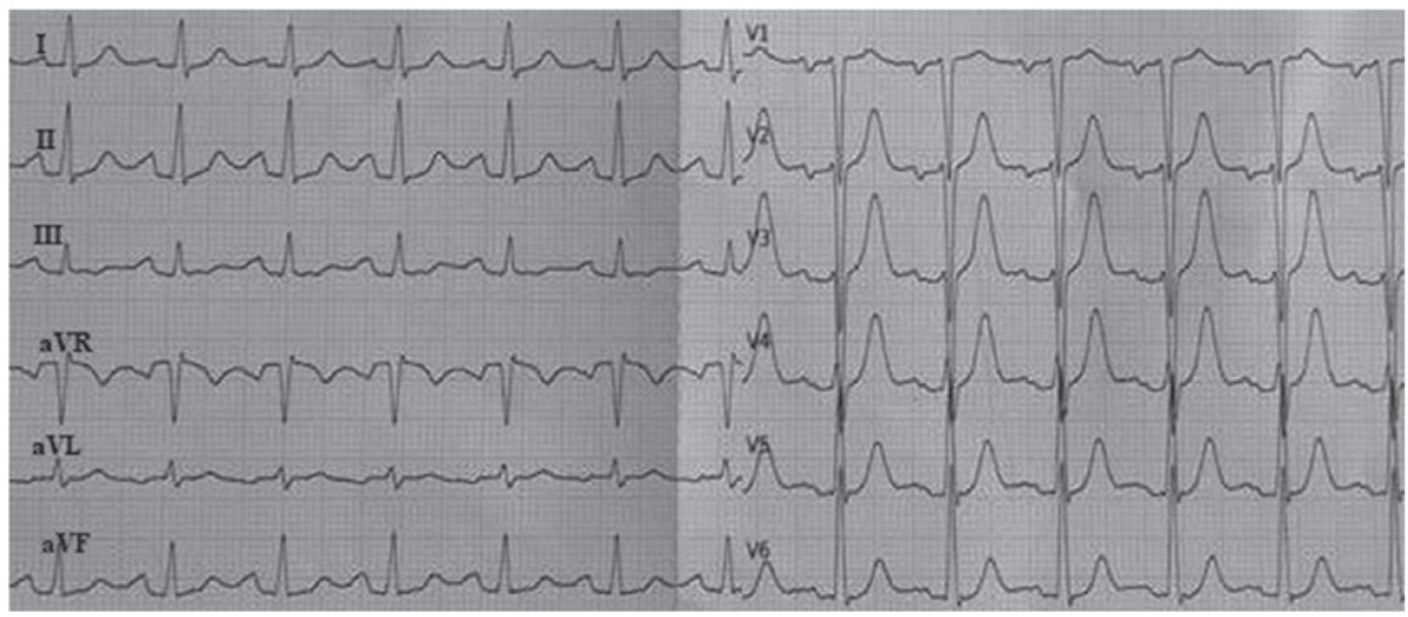

Figure 3. 12-leads electrocardiogram of an asymptomatic patient (age, 32 years). The Sokolow index is in the normal range. Normal regular sinus rhythm with hyperkalemia, which caused left ventricular hypertrophy. No sign of angina. I, II, III, V1, V2, V3, V4, V5 and V6 are external leads. aVR, augmented vector right; aVL, augmented vector left; aVF, augmented vector foot.

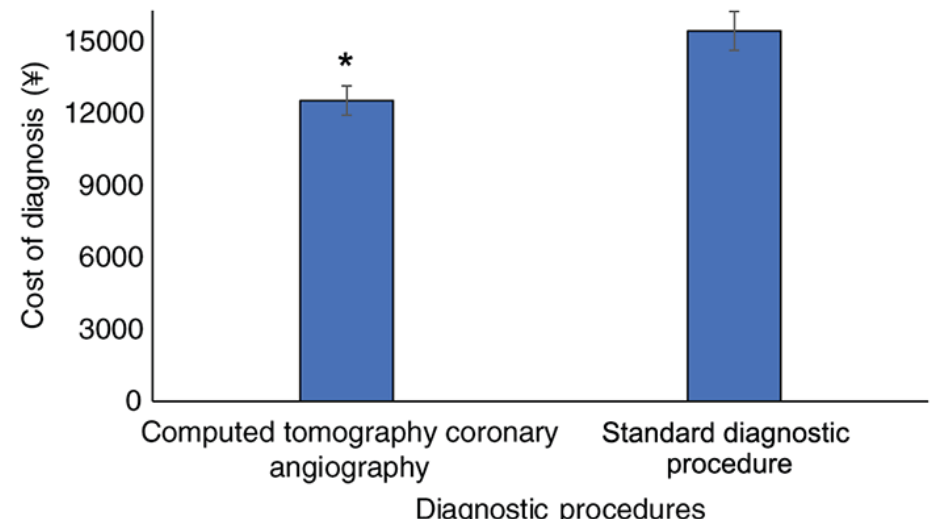

Figure 4. Cost of a diagnostic procedure for detection of angina due to coronary heart disease. One-way analysis of variance was performed for statistical analysis. $\mathrm{P}<0.01$ was considered to indicate a significant difference. $6 ¥ \equiv 1$ US $\$$. The 'standard diagnostic procedure’ included clinical assessments, a $12-$ lead electrocardiogram and an exercise electrocardiogram. ${ }^{*} \mathrm{P}<0.05$ vs. standard diagnostic procedure.

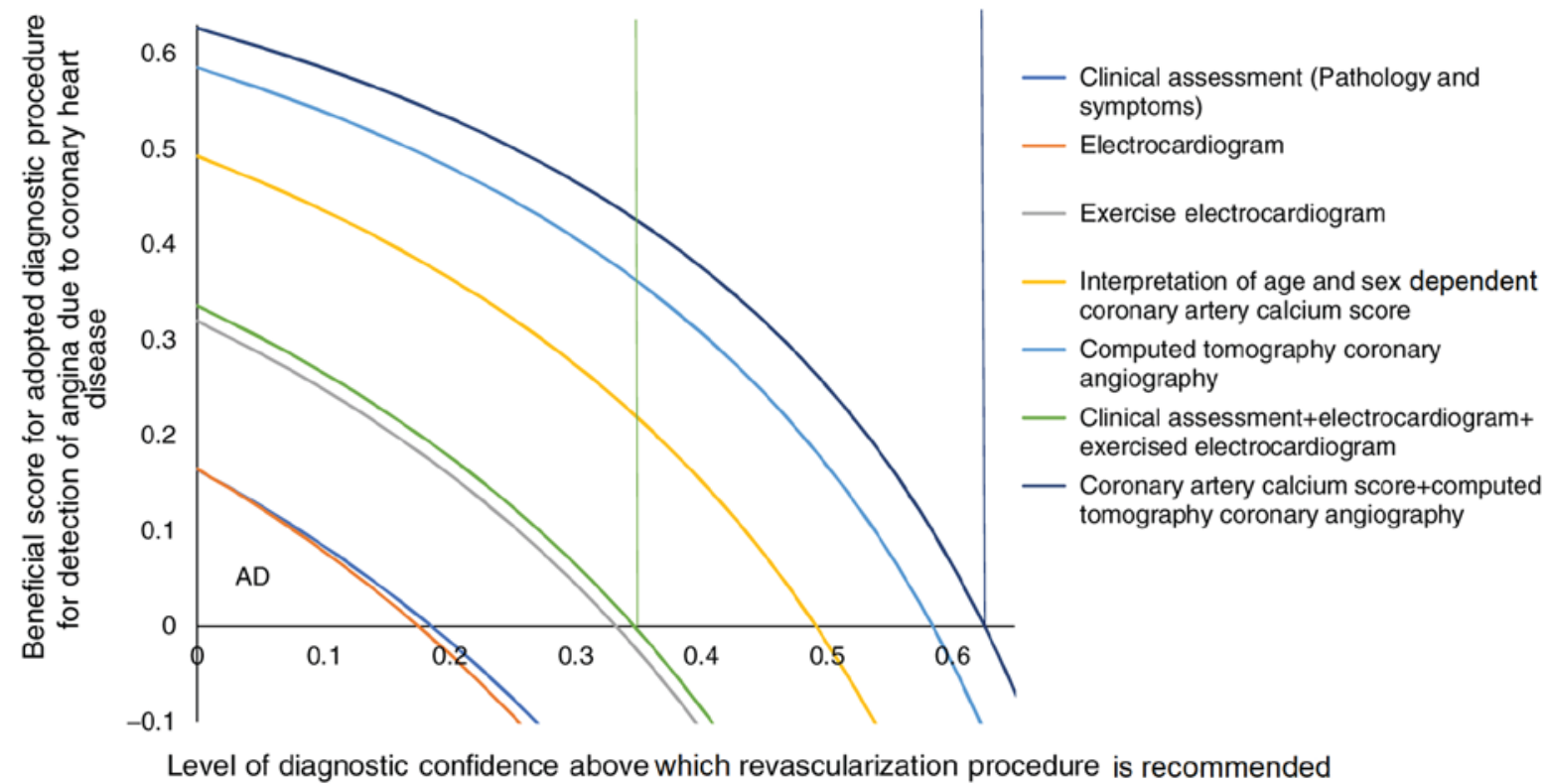

Figure 5. Benefit score curves for adopted diagnostic procedures. AD, the working area of the diagnosis detected angina due to coronary heart disease at one time. The 'standard diagnostic procedure' included clinical assessments, a 12-lead electrocardiogram and an exercise electrocardiogram. 
The cost of diagnosis using the standard diagnostic procedure was higher than that of CTCA, as the former includes clinical assessments, ECG and exercise ECG. Pathology includes several types of tests, which are costly, time-consuming and tedious. ECG is first-line test and international guidelines also suggested this test for any possible angina due to heart diseases (12). In consideration of cost factors, exercise ECG increases the undesired financial burden on low- and moderate-income patients.

Regarding the limitations of the present study, the sensitivity and specificity of the diagnostic tests compared with a reference standard (invasive CA) should have been assessed, which is lacking in the present study. Without a reference standard, it may be wrongly assumed that the best diagnostic test is the one detecting the most anomalies in the population of the study. Furthermore, it may not be appropriate to perform a head-to-head comparison of CTCA, which exposes patients to radiation, with other imaging modalities. In addition, the present study did not report on cardiac events in the patients after diagnosis. The present study has also not discussed the use of the diagnostic methods to guide the selection of these drugs ( $\beta$-blockers and/or glyceryl trinitrate) in the follow-up period. The limited applicability of coronary artery calcium score (45-84 years) is a further limitation. The cost factor is not generalized and applies to the P.R. China only. A huge number of patients arrived at the hospital during the study period and were managed/treated by large number of medical and paramedical staff; therefore, intra- and inter-observer reliabilities are not granted. The study also lacks subgroup analyses to identify any possible advantages of CTCA in patients with typical or atypical chest pain.

The present study included 2,426 patients with chest pain referred to the rapid access chest pain clinic of Beijing Anzhen Hospital, Capital Medical University (Beijing, China). It may be concluded that the diagnostic cost and the duration of the hospital stay per individual are less for CTCA than those for the 'standard diagnostic procedure'. The present study provided useful information that may enhance the current knowledge regarding CTCA and 'standard diagnostic procedure' for the diagnosis of angina due to coronary heart disease. However, whether all chest pain patients require CTCA examination remains controversial, particularly for those with atypical chest pain. After all, the potential risk associated with CTCA is higher than that of clinical assessments, ECG and exercise ECG. Overall, the present study is of significance for non-acute coronary syndrome patients presenting at chest pain clinics, and may provide guidance on what diagnostic modality to perform first, CTCA or 'standard diagnostic procedure'.

In conclusion, according to the beneficial score analysis curve, CTCA had a higher sensitivity for the diagnosis of angina due to coronary heart disease and the cost was lower than of the 'standard diagnostic procedure' diagnosis. Based on the results of the present study, it may be recommended to only perform CTCA in patients with suspected angina referred to a chest pain clinic and to not subject them to the other stressful imaging modalities and tedious pathological examination.

\section{Acknowledgements}

Not applicable.

\section{Funding}

No funding was received.

\section{Availability of data and materials}

The datasets used and/or analyzed during the present study are available from the corresponding author on reasonable request.

\section{Authors' contributions}

All authors read and approved the manuscript prior to submission. ZW contributed to the design of the study and project administration. YH contributed to data curation and formal analysis. WL performed the statistical analysis. SC contributed to data curation, and drafted, reviewed and edited the manuscript for intellectual content.

\section{Ethics approval and informed consent}

The protocol of the present study (no. BAH/CL/01/16 dated 15 December 2015) was approved by the review board of Beijing Anzhen Hospital (Beijing, China).

\section{Patient consent for publication}

Not applicable.

\section{Competing interests}

The authors declare that they have no competing interests.

\section{References}

1. Williams MC, Hunter A, Shah A, Assi V, Lewis S, Mangion K, Berry C, Boon NA, Clark E, Flather M, et al: Symptoms and quality of life in patients with suspected angina undergoing CT coronary angiography: A randomised controlled trial. Heart 103: 995-1001, 2017

2. Bennell MC, Qiu F, Kingsbury KJ, Austin PC and Wijeysundera HC: Determinants of variations in initial treatment strategies for stable ischemic heart disease. CMAJ 187: E317-E325, 2015.

3. Jordan KP, Timmis A, Croft P, van der Windt DA, Denaxas S, González-Izquierdo A, Hayward RA, Perel P and Hemingway H: Prognosis of undiagnosed chest pain: Linked electronic health record cohort study. BMJ 357: j1194, 2017.

4. Sekhri N, Feder GS, Junghans C, Hemingway H and Timmis AD: How effective are rapid access chest pain clinics? Prognosis of incident angina and non-cardiac chest pain in 8762 consecutive patients. Heart 93: 458-463, 2007.

5. Boyle RM: Value of rapid-access chest pain clinics. Heart 93: 415-416, 2007.

6. Parsonage WA, Cullen L and Younger JF: The approach to patients with possible cardiac chest pain. Med J Aust 199: 30-34, 2013.

7. Nishimura RA, Otto CM, Bonow RO, Carabello BA, Erwin JP III, Fleisher LA, Jneid H, Mack MJ, McLeod CJ, O'Gara PT, et al: 2017 AHA/ACC focused update of the 2014 AHA/ACC guideline for the management of patients with valvular heart disease: A report of the American College of Cardiology/American Heart Association Task Force on Clinical Practice Guidelines. J Am Coll Cardiol 70: 252-289, 2017.

8. Sajjadieh A, Hekmatnia A, Keivani M, Asoodeh A, Pourmoghaddas $M$ and Sanei H: Diagnostic performance of 64-row coronary $\mathrm{CT}$ angiography in detecting significant stenosis as compared with conventional invasive coronary angiography. ARYA Atheroscler 9: 157-163, 2013 
9. Dreisbach JG, Nicol ED, Roobottom CA, Padley S and Roditi G: Challenges in delivering computed tomography coronary angiography as the first-line test for stable chest pain. Heart 104 921-927, 2018

10. Mowatt G, Cummins E, Waugh N, Walker S, Cook J, Jia X, Hillis GS and Fraser C: Systematic review of the clinical effectiveness and cost-effectiveness of 64-slice or higher computed tomography angiography as an alternative to invasive coronary angiography in the investigation of coronary artery disease. Health Technol Assess 12: iii-iv, ix-143, 2008.

11. Fuchs TA, Stehli J, Bull S, Dougoud S, Clerc OF, Herzog BA, Buechel RR, Gaemperli O and Kaufmann PA: Coronary computed tomography angiography with model-based iterative reconstruction using a radiation exposure similar to chest X-ray examination. Eur Heart J 35: 1131-1136, 2014.

12. Hamilton-Craig C, Fifoot A, Hansen M, Pincus M, Chan J, Walters DL and Branch KR: Diagnostic performance and cost of CT angiography versus stress ECG-a randomized prospective study of suspected acute coronary syndrome chest pain in the emergency department (CT-COMPARE). Int J Cardiol 177: 867-873, 2014.

13. Cohen JF, Korevaar DA, Altman DG, Bruns DE, Gatsonis CA, Hooft L, Irwig L, Levine D, Reitsma JB, de Vet HCW and Bossuyt PMM: STARD 2015 guidelines for reporting diagnostic accuracy studies: Explanation and elaboration. BMJ Open 6 : e012799, 2016

14. World Medical Association: World medical association declaration of helsinki: Ethical principles for medical research involving human subjects. JAMA 310: 2191-2194, 2013

15. Arnold J, Goodacre S, Bath P and Price J: Information sheets for patients with acute chest pain: Randomised controlled trial. BMJ 338: b541, 2009.

16. Newby DE, Williams MC, Flapan AD, Forbes JF, Hargreaves AD, Leslie SJ, Lewis SC, McKillop G, McLean S, Reid JH, et al: Role of multidetector computed tomography in the diagnosis and management of patients attending the rapid access chest pain clinic, The Scottish computed tomography of the heart (SCOT-HEART) trial: Study protocol for randomized controlled trial. Trials 13: 184, 2012.

17. Timmis A and Roobottom CA: National institute for health and care excellence updates the stable chest pain guideline with radical changes to the diagnostic paradigm. Heart 103: 982-926, 2017.

18. Lennon SL, DellaValle DM, Rodder SG, Prest M, Sinley RC, Hoy MK and Papoutsakis C: 2015 Evidence analysis library evidence-based nutrition practice guideline for the management of hypertension in adults. J Acad Nutr Diet 117: 1445-1458.e17, 2017.

19. Kim KH, Jeon KN, Kang MG, Ahn JH, Koh JS, Park Y, Hwang SJ, Jeong YH, Kwak CH, Hwang JY and Park JR: Prognostic value of computed tomographic coronary angiography and exercise electrocardiography for cardiovascular events. Korean J Intern Med 31: 880-890, 2016
20. National Institute for Health and Clinical Excellence: Guidance: Chest pain of recent onset: Assessment and diagnosis of recent onset chest pain or discomfort of suspected cardiac origin [Internet]. National Clinical Guideline Centre for Acute and Chronic Conditions (UK). London: Royal College of Physicians (UK), 2010

21. Fitzgerald M, Saville BR and Lewis RJ: Decision curve analysis. JAMA 313: 409-410, 2015.

22. Blaha MJ, Budoff MJ, Tota-Maharaj R, Dardari ZA, Wong ND, Kronmal RA, Eng J, Post WS, Blumenthal RS and Nasir K: Improving the CAC score by addition of regional measures of calcium distribution: Multi-ethnic study of atherosclerosis. JACC Cardiovasc Imaging 9: 1407-1416, 2016.

23. George RT, Arbab-Zadeh A, Miller JM, Kitagawa K, Chang HJ, Bluemke DA, Becker L, Yousuf O, Texter J, Lardo AC and Lima JA: Adenosine stress 64- and 256-row detector computed tomography angiography and perfusion imaging: A pilot study evaluating the transmural extent of perfusion abnormalities to predict atherosclerosis causing myocardial ischemia. Circ Cardiovasc Imaging 2: 174-182, 2009.

24. Holt T: Chest pain in primary care: What happens to the undiagnosed majority? BMJ 357: j1626, 2017.

25. Xu Y, Tang L, Zhu X, Xu H, Tang J, Yang Z, Wang L and Wang D: Comparison of dual-source CT coronary angiography and conventional coronary angiography for detecting coronary artery disease. Int J Cardiovasc Imaging 26 (Suppl 1): S75-S81, 2010.

26. SCOT-HEART Investigators: CT coronary angiography in patients with suspected angina due to coronary heart disease (SCOT-HEART): An open-label, parallel-group, multicentre trial. Lancet 385: 2383-2391, 2015.

27. Sabaté M and Ishida K: CT coronary angiography increase diagnostic certainty in patients with stable chest pain. Evid Based Med 20: 187, 2015

28. Boutron I, Moher D, Altman DG, Schulz KF and Ravaud P; CONSORT Group: Extending the CONSORT statement to randomized trials of nonpharmacologic treatment: Explanation and elaboration. Ann Intern Med 148: 295-309, 2008.

29. US and Food and Drug Administration. Step 3. Clinical research. https://www.fda.gov/ForPatients/Approvals/Drugs/ucm405622. htm. (Accessed on 15 October, 2016).

This work is licensed under a Creative Commons Attribution-NonCommercial-NoDerivatives 4.0 International (CC BY-NC-ND 4.0) License. 